Proc. 13th Econophysics Colloquium (EC) and 9th Symposium of Physics in Economy and Social Sciences (FENS), 2017

\title{
Self-Organization of Extreme Inequalities in a Competitive Society
}

\begin{abstract}
T. ODAGAKI ${ }^{a, *}$ AND A. ISHIFUKU ${ }^{b}$
${ }^{a}$ Research Institute for Science Education, Inc. 8 Tojiin Kitamachi, Kitaku, Kyoto 603-8346, Japan

${ }^{b}$ School of Engineering and Science, Tokyo Denki University Hatoyamacho, Saitama 350-0394, Japan

On the basis of a random walk model, we investigate the self-organization of inequality in a model competitive society which consists of two kinds of individuals; one is warlike-challenging individuals who always try to fight and fight with the wealthiest or strongest neighbor, and the other is pacific-timid individuals who always try not to fight and when necessary fight with the poorest or weakest neighbor. When two individuals meet on a lattice site, they fight and the winner deprives a unit wealth from the loser keeping its position, where the winning odd is determined by a sigmoid function of the difference in their wealths. At the same time, the wealth or debt of individuals relaxes to zero at a constant rate when the wealth or debt is large. Using Monte Carlo simulation we determine states of social inequality in the entire parameter space spanned by the population density and the fraction of pacific-timid individuals in the population on the basis of the profile of the wealth distribution plotted against the ranking. We find an egalitarian state, and one normal inequal and three different extreme inequal states, the plutonomy, the gap inequality and the terrace inequality. In order to elucidate the origin of the self-organization, we investigate a model society consisting of individuals who have different moving strategies and no specific fighting strategy. It is concluded that the extreme inequalities are the consequence of the coexistence of different fighting strategies.
\end{abstract}

DOI: 10.12693 /APhysPolA.133.1459

PACS/topics: 05.65.+b, 89.65.Gh

\section{Introduction}

In the modern world, economically advanced nations have been suffering emergence of inequality in their society. In the fall of 2011, a big demonstration occupied Wall Street in New York City and the traders in Wall Street were blamed for causing the plutonomy, or an extreme form of inequal society [1, 2]. It is puzzling why the plutonomy has emerged in the US where free and fair competitions are encouraged [3]. Although this trend has also been observed in Canada and Great Britain [4, 5], inequality in Japan seems to be of a different form. The Annual Report on the Japanese Economy and Public Finance 2009 reported by Cabinet Office of the Japanese government [6] showed that a different form of inequality has been enhancing in Japan. The data presented in the report indicates that number of people in the middle class has been reduced and that in the lower class has been increasing as if the society is going to be divided into winners and losers without the middle class [7].

On the basis of detailed analysis of economic data for more than 100 years, Piketty [8] argued that when the rate of return on capital is greater than the rate of economic growth over the long term, wealth concentrates to a small number of people and this unequal distribution of wealth causes social inequality. This argument, however, cannot explain the self-organization of different forms of extreme inequality. Furthermore, selforganization of inequality or hierarchy has been observed

* corresponding author; e-mail: t.odagaki@kb4.so-net.ne.jp in social animals such as Japanese monkeys and chickens and in social insects such as paper wasps. Therefore, the self-organization of inequality must be a universal phenomenon in competitive societies, and thus there must be a universal mechanism of the self-organization. It is the responsibility of physics to unveil the universal mechanism and to find parameters of the society which lead to different inequal states, in the same way as the phase transitions are understood by statistical mechanics.

Bonabeau et al. [9] first proposed a simple model (referred to as the random walk model) for a competitive society consisting of random walkers and showed that the inequality is self-organized when the frequency of fighting between individuals exceeds a critical value. At the critical value, winners and losers begin to appear together with individuals in the middle class. As the frequency is increased further, the middle class diminishes and the wealth profile plotted against ranking becomes a straight line. We call this state as the normal inequality. Fujie and Odagaki [10] introduced a model society (referred to as the random participation model) where individuals participate in fighting without making the random walk and showed that the structure in inequal states strongly depends on the cost and reward of the fighting. Therefore, inequality can be self-organized in competitive societies where (1) an individual fights with an opponent at a given frequency and the wealthier or stronger has a higher probability to win the fight, (2) the winner deprives the loser of its wealth, and (3) the wealth or debt (negative wealth) of an individual relaxes to zero at a constant rate when the wealth or debt is large. When the frequency of fights exceeds a critical value, the transfer of wealth occurs more frequently than the relaxation rate 
of the wealth, and winners who keep winning and losers who keep losing appear in the society, leading to an inequal state. In the inequal state, although winners, losers and middle class can be identified, the wealth of individuals changes rather regularly as a function of their ranking and is different from that seen in plutonomic society, where the number of the winners is a small fraction of the population and the wealth keeps accumulating in the winners. It is also completely different from the inequality in Japan characterized by missing middle class [6].

Odagaki and Tsujiguchi [11, 12], Okubo and Odagaki [13] and Fujie and Odagaki [14] reported within the random walk model that the self-organized inequal state depends strongly on the strategy of individuals in the random walk and fighting. Recently Todate et al. [15] showed that a fair competition mechanism in the random participation model induces the plutonomy, where individuals grouped into several classes compete with those in the same class for a certain period (season) and they are regrouped at the end of every season [15].

Several other models have been proposed to explain the emergence of hierarchies $[16,17]$.

In this paper, we consider a model society which consists of two kinds of individuals making random walk on a square lattice; one is warlike-challenging (WC) individuals who always try to fight and when possible fight with the strongest neighbor, and the other is pacific-timid (PT) individuals who always try not to fight and when necessary fight with the weakest neighbor. We investigate the self-organization of inequality in this model society by Monte Carlo simulation. We analyze the structure of social inequality in the entire parameter space spanned by the population density $\rho$ and the fraction $x$ of pacifictimid individuals in the population. Basically, we can use the fluctuation in the winning probability of all individuals as an order parameter [9]. However, the behavior of the fluctuation is rather subtle and sometime misleading as an order parameter [14], and thus we exploit the profile of wealth distribution of all individuals in order to identify different states of inequality. We find an egalitarian state, and one normal inequal and three different extreme inequal states which are the plutonomy, the gap inequality and the terrace inequality.

In order to get insight for the self-organization of different extreme inequalities, we investigate a different model society which consists of war-like individuals and pacifist individuals without challenging or timid nature. We find only the normal inequal states in the entire parameter space, and thus it is concluded that the extreme inequalities are the consequence of coexistence of two kinds of fighting strategies.

We organize this paper as follows. In Sect. 2, we explain our model society in detail and the method of Monte Carlo simulation. In Sect. 3.1, we show first how the profile of the wealth distribution changes when the population density is increased for $x=0.5$. The phase diagram in the entire parameter space is presented in Sect. 3.2. In Sect. 4, we discuss the importance of chal- leging and timid natures by investigating the different model society. We give a brief discussion in Sect. 5 .

\section{Model and Monte Carlo simulation}

We consider a society of $N$ individuals who make a one step random walk at each Monte Carlo step in a random order on an $L \times L$ square lattice with periodic boundary conditions. The wealth of individual $i$ is denoted by $F_{i}$ which determines the strength of individual $i$. When individual $i$ moves on a site occupied by individual $j$, they fight and the winner is randomly deteremined by the winning odd of individual $i$ against individual $j$, $W_{i j}$, given by

$$
W_{i j}=\frac{1}{1+\exp \left[\eta\left(F_{i}-F_{j}\right)\right]},
$$

where $\eta(>0)$ is a control parameter of the model. The winner gets a unit of wealth and the loser loses a unit of wealth. As for the position, the winner takes the site and the loser is forced to move to the site occupied by individual $i$ before the step. This winning odd (1) ensures that the wealthier (or stronger) has a higher probability of winning. The wealth of individuals is assumed to decay at every Monte Carlo step as follows:

$$
F_{i}(t+1)=F_{i}(t)-\mu \tanh \left(F_{i}(t)\right)
$$

that is, when $\left|F_{i}(t)\right|$ is small, it decays with a rate in proportion to $F_{i}(t)$ :

$$
F_{i}(t+1)=(1-\mu) F_{i}(t),
$$

and when $\left|F_{i}(t)\right|$ is large, it decays or increases at a constant rate

$$
F_{i}(t+1)=F_{i}(t)-\mu \operatorname{sgn}\left(F_{i}(t)\right) .
$$

In this research, we introduce two kinds of individuals, each of which has different moving and fighting strategies. Table I summarizes the two kinds of individuals.

TABLE I

The moving and fighting strategies of pacific-timid (PT) individuals and warlike-challenging (WC) individuals

\begin{tabular}{c|c|c}
\hline \hline & $\begin{array}{c}\text { Pacific } \\
\text { timids (PT) }\end{array}$ & $\begin{array}{c}\text { Warlike } \\
\text { challengers (WC) }\end{array}$ \\
\hline $\begin{array}{c}\text { moving } \\
\text { strategy }\end{array}$ & $\begin{array}{c}\text { moves onto } \\
\text { a vacant site, if any }\end{array}$ & $\begin{array}{c}\text { moves onto } \\
\text { an occupied site, if any }\end{array}$ \\
\hline $\begin{array}{c}\text { fighting } \\
\text { strategy }\end{array}$ & $\begin{array}{c}\text { fight } \\
\text { with the weakest }\end{array}$ & $\begin{array}{c}\text { challenge } \\
\text { to the strongest }\end{array}$ \\
\hline fraction & $x$ & $1-x$
\end{tabular}

A PT individual moves to a vacant site if it exists and when there is no vacant site in the neighbors, it attacks the weakest one in the neighbors. A WC individual moves onto a site occupied by some other individual if any, and when there are more than two occupied sites, it always challenges to the strongest one. We denote by $x$ the fraction of $\mathrm{PT}$ individuals in the population, and thus the fraction of $\mathrm{WC}$ individuals is $1-x$. 
The self-organization of inequality in the society for $x=0$ and $x=1$ has been studied. For the society of $x=1$, the self-organization occurs in two steps, the critical density is much higher than one for Monabeau's simple model, and at the first transition, losers appears and at the second transition large number of winners appear $[11,13,14]$. For the society of $x=0$, the critical density for the self-organization is smaller than that for the simple model, and villages are formed in the inequal society [12] and the plutonomy emerges when each idividual makes a one step random walk in random order and the population density is high. Our aim is to find the phase diagram in the entire space spanned by the population density $\rho=\frac{N}{L \times L}$ and $x$.

We performed Monte Carlo simulation for a society of $N=2500$ individuals who make one step random walk in random order on an $L \times L$ square lattice with the periodic boundary conditions. $\rho$ is controlled by changing the lattice size $L$. We set $\eta=5.0$ and $\mu=0.1$ for the present simulation, and results are shown for the steady state reached after $3 \times 10^{6}$ Monte Carlo steps.

\section{Self-organization of various inequal states}

\subsection{Emergence of inequality at $x=0.5$}

We first present how the various inequal states develop as a function of the population density when the fractions of two species are equal, i.e. $x=0.5$. In Fig. 1, we plot the wealth of individuals against its ranking for four different population densities; (a) $\rho=0.02$, (b) $\rho=0.1$, (c) $\rho=0.4$ and (d) $\rho=0.88$. We also show in the insets of each figure the wealth profiles of the $\mathrm{WC}$ and the PT individuals. When $\rho$ is small, the width of the wealth distribution is small and temporal, and the ranking of individuals are not fixed. Therefore the society is in the egalitarian state and PT and WC individuals are distributed evenly in the ranking. The profile of the distribution of wealths shown in Fig. 1b shows the typical inequal society which is seen in the normal competitive society [9]. Namely the society consists of winners who keep winning, losers who keep losing and the middle class whose ranking changes continually. The state shown in Fig. 1c corresponds to the plutonomy where the winners, who are some of the WC individuals, are only a small fraction of population, and most of population are in the middle class. The inequal society Fig. 1d has a characteristic profile of the wealth distribution which misses the middle class. We call this state the gap inequality.

It is interesting to note that the distribution of $\mathrm{WC}$ and PT individuals in the ranking is not common but takes different forms depending on the structure of inequality. In the normal inequal state shown in Fig. 1b, the WC individuals belong to the winners or the losers and the middle class consists only of the PT individuals. The fraction of the WC individuals in the winners becomes smaller in the plutonomic state shown in Fig. 1c and almost disappears in the gap inequal state shown in
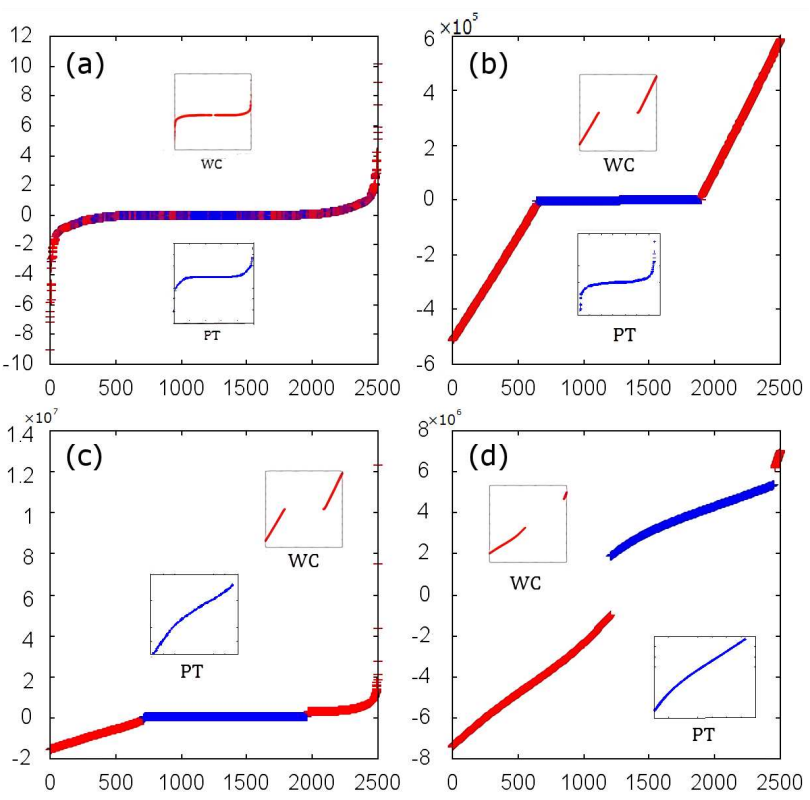

Fig. 1. The wealth profile of all individuals as a function of their ranking: (a) $\rho=0.02$, (b) $\rho=0.1$, (c) $\rho=0.4$ and (d) $\rho=0.88$ at $x=0.5$. The insets of each figure show the wealth profiles of the WC and the PT individuals. Note that the vertical scales are different and that the scales of the insets are set so that the details can be seen.

Fig. 1d. In the gap inequal state Fig. 1d, the PT individuals become winners and most of the WC individuals are the losers.

\subsection{Phase diagram in the entire parameter space}

We examined the wealth profile for every $\rho=0.01 \times$ $n(n=1,2, \ldots, 100)$ at every $x=0.1 \times m(m=$ $0,1,2, \ldots, 10)$, focusing on the following points:

(1) if there is an individual whose wealth or debt increases with time.

(2) if there exist winners, losers, and middle class, and the dependence of the wealth (debt) of winners (losers) on the ranking is a straight line.

(3) if there is only a small fraction of winners.

(4) if there is a gap in wealths for winners and losers. and

(5) if there are many winners at almost the same level of wealth.

With careful observation of the wealth profile for $100 \times 11$ mesh points on the $\rho-x$ plane, we determined the phase diagram on the $\rho-x$ plane. Figure 2 shows the phase diagram of the society, where five different phases are identified, and the typical wealth profiles of each phase are shown in Fig. 3. We concluded that the five different phases are: (1) the egalitarian state, (2) the normal inequal state, (3) the plutonomic state, (4) the gap inequal state and (5) a state which consists of many winners at the same wealth level and many losers who are distributed in a wide range of wealth. While 
states (1)-(4) are the same as described in Sect. 3.1, the state (5) is a new state which may be called the terrace inequality. Note that the error bars for the phase boundaries in Fig. 2 are \pm 0.005 in the vertical $\rho$ direction and \pm 0.05 in the horizontal $x$ direction. As shown in Fig. 3e, the terrace inequal state consists of a large number of winners at almost the same wealth level which are the PT individuals and the other individuals whose wealth decrease gradually towards the lowest ranking which are mostly the $\mathrm{WC}$ individuals.

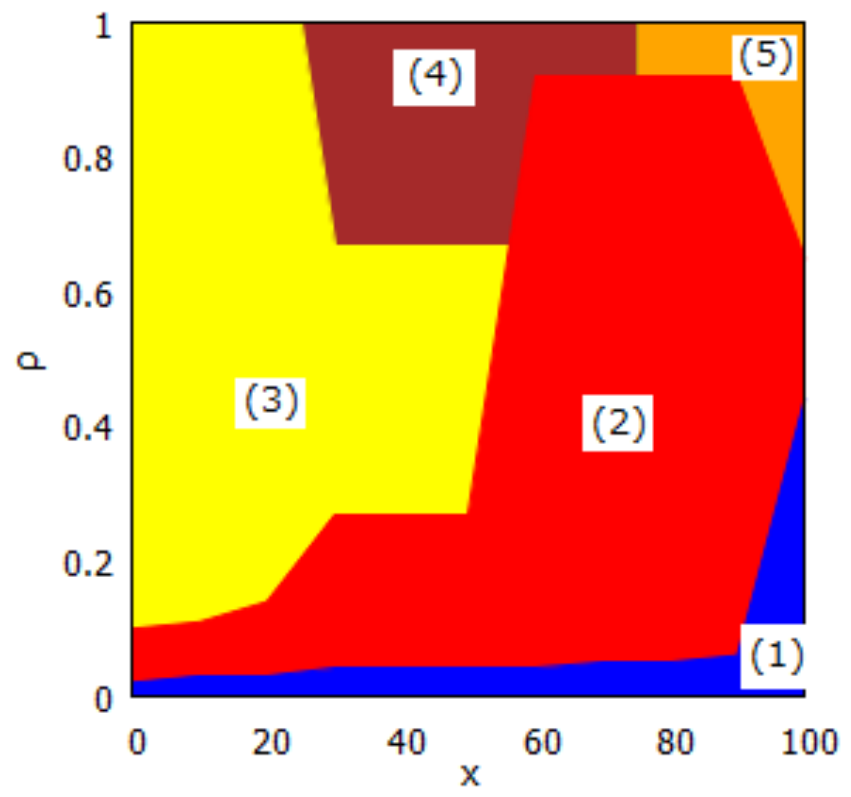

Fig. 2. The phase diagram in the entire parameter space: (1) the egalitarian state, (2) the normal-inequal state, (3) the plutonomic state, (4) the gap-inequal state and (5) the terrace-inequal state.

\section{Origin of the extreme inequalities}

In order to elucidate the origin of the extreme inequalities, we investigated a society consisting of warlike individuals without the challenging strategy and pacifist individuals without the timid nature. Namely, all individuals do not have any strategy in choosing an opponent when needed. Table II summarizes the two kinds of individuals.

TABLE II

The moving and fighting strategies of war-like (W) individuals and pacific $(\mathrm{P})$ individuals.

\begin{tabular}{c|c|c}
\hline \hline & Pacifists (P) & Warlikes (W) \\
\hline $\begin{array}{c}\text { moving } \\
\text { strategy }\end{array}$ & $\begin{array}{c}\text { move onto } \\
\text { a vacant site, if any }\end{array}$ & $\begin{array}{c}\text { move onto } \\
\text { an occupied site, if any }\end{array}$ \\
\hline $\begin{array}{c}\text { fighting } \\
\text { strategy }\end{array}$ & random & random \\
\hline fraction & $x$ & $1-x$
\end{tabular}

Figure 4 shows the phase diagram of this society in the entire parameter space spanned by $\rho$ and $x$, where
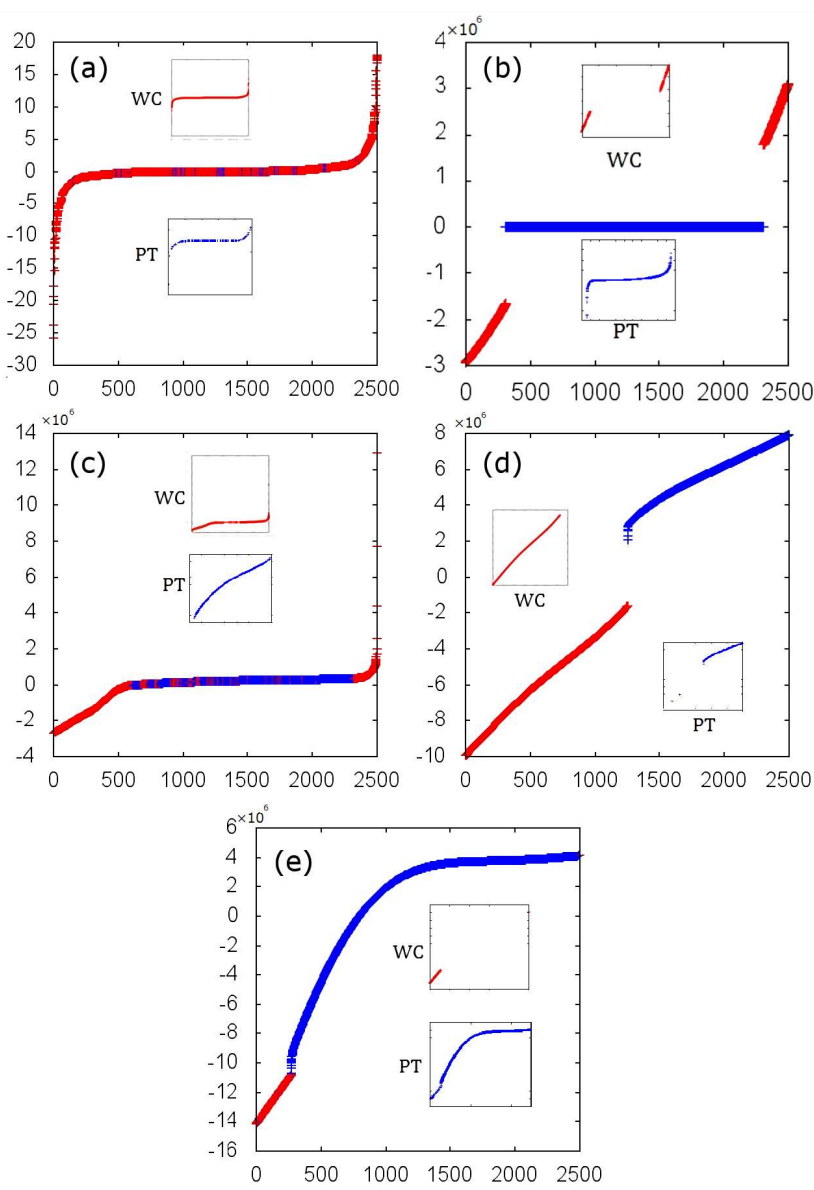

Fig. 3. The typical wealth profile in each state shown in Fig. 2. The parameters $(\rho, x)$ are (a) $(0.01,0.1)$ in the egalitarian state (1), (b) $(0.48,0.8)$ in the normalinequal state $(2),($ c) $(0.57,0.3)$ in the plutonomic state (3), (d) $(1.0,0.5)$ in the gap-inequal state $(4)$, and (e) $(1.0,0.9)$ in the terrace-inequal state $(5)$. Note that the vertical scales are different and that the scales of the insets are set so that the details can be seen. (WC and PT individuals are shown by blue and red symbols, respectively, in color figure.

$100 \times 11$ points in the $\rho-x$ plane as in Fig. 2, and therefore the error bars for the phase boundaries are \pm 0.005 in the vertical $\rho$ direction and \pm 0.05 in the horizontal $x$ direction. Figure $5 \mathrm{a}$ and $\mathrm{b}$ shows the typical wealth profiles for states (3) and (4) defined in Fig. 4. The wealth profile for states (1) and (2) in Fig. 4 are identical to those shown in Fig. $3 a$ and b. When the population density is low, the society is in the egalitarian state (1) and at a critical population the normal inequal state (2) emerges. When the population density is increased further, inequality emerges in the middle class (3) and eventually the society becomes a state (4) where the wealth vs. ranking plot becomes a strait line.

This result indicates that the extreme forms of inequality, the plutonomy, the gap inequality and the terrace inequality, are self-organized by coexistence of different 


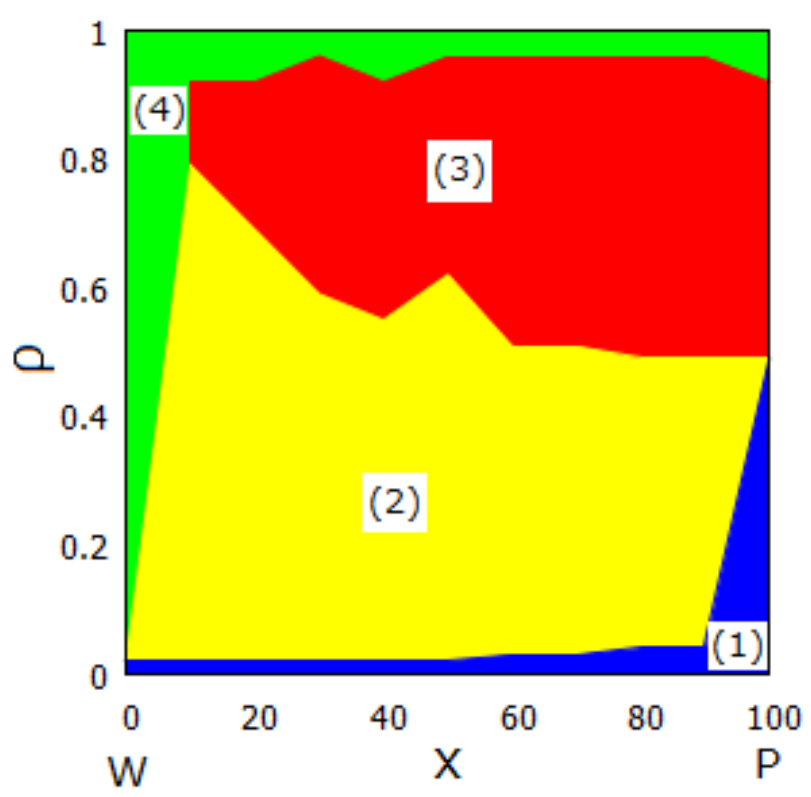

Fig. 4. The phase diagram in the entire parameter space for the society consisting of the $\mathrm{W}$ and $\mathrm{P}$ individuals. (1) the egalitarian state, and (2)-(4) are the normal-inequal state with different type wealth distribution.
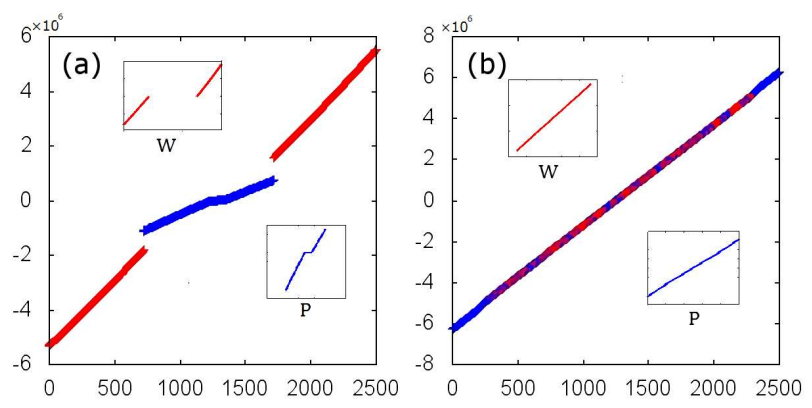

Fig. 5. The typical wealth profile in states 3 and 4 in Fig. 4. The parameters $(\rho, x)$ are (a) $(0.82,0.5)$ in the state (3) and (b) $(1.0,0.5)$ in the state (4), Note that the vertical scales are different and that the scales of the insets are set so that the details can be seen..

fighting strategies, i.e. the challenging and timid strategies. In particular, when the fraction of the WC individuals is high, a layer structure of individuals in the order of wealth is formed with the wealthiest one at the center [14] and the wealth flows continually from the less wealthiers to the wealthiers. This structure is the origin of the plutonomy. The gap inequal state emerges when the weak challengers challenge to stronger timid individuals and strong timid individuals always fight with weak challenging individuals.

\section{Discussion}

We have investigated the self-organization of various extreme inequalities in a simple competitive soci- ety where all individuals make random walk on a square lattice and wealth of an individuals changes by fighting with other individual and by relaxation. We introduced two different groups of individuals characterized by their moving and fighting strategies.

It is shown that there are three types of extreme inequality: the plutonomy, the gap inequality and the terrace inequality. When the fraction of $\mathrm{WC}$ individuals is high, a layer structure is formed and the wealth continually flows from the weakers to the strongers and the plutonomy is self-organized. When the fraction of PT individuals is high, some of PT keep winning by attacking weakers and form a winning group and the terrace inequality is self-organized. When both PT and WC individuals exist in some fractions, the strong PT individuals keep winning by attacking the weak WC individuals and the weak WC individuals keep losing by challenging the strong PT individuals, and the gap inequality is self-organized.

The present results indicate that extreme inequal societies can be self-organized just by competition among individuals, and that the strategy of individuals plays important role in the self-organization. Namely, the challengers are likely to fight with a stronger and loses. The timids as a matter of fact fight with weakers and are likely to win when the population density is high.

We can think of pacifists with the challenging nature (PC individuals). By referring Okubo nd Odagaki [13], we can expect that the society consisting of the $\mathrm{WC}$ individuals and the $\mathrm{PC}$ individuals will show an inverseterrace inequality when the population density is high, where many losers at the same debt level appear together with individuals whose wealth increase gradually towards the highest ranking.

For the pure PT society $x=1$, Okubo and Odagaki [13] reported an analysis based on the mean-field approximation and showed that many winners at the same wealth level emerge at an upper critical value of $\rho$. This is, thus, the origin of the terrace extreme inequality in the present model. The mean field analysis, however, cannot be applied for $x<1$, where WC individuals tend to produce spatially inhomogeneous structure. It is a challenging open problem to obtain the more accurate phase diagram theoretically in the entire parameter space of the present model.

In passing, it is interesting to compare the selforganization of inequality with phase transitions where strong interaction among constituents leads to various phases. The self-organization of social structures such as inequality and segregation is driven by strategies of individuals for moving and/or some other activities [18].

\section{Acknowledgments}

We would like to thank Mr. K. Kitada and Mr. K. Omizo for their contribution in the early stage of the present work. 


\section{References}

[1] Occupy!: Scenes from Occupied America, Eds. C. Blumenkranz, K. Gessen, M. Greif, S. Leonard, S. Resnick, Verso Books, 2011.

[2] G. Chin, E. Culotta, Science 344, 818 (2014).

[3] Congressional Budget Office, The Distribution of Household Income and Federal Taxes, 2010, Washington, D.C. 2013.

[4] A.B. Atkinson, J. Hasell, S. Morelli, M. Roser, The Chartbook of Economic Inequality, 2017.

[5] F. Alvaredo, T. Atkinson, T. Piketty, E. Saez, The World Wealth and Income Database, 2017.

[6] Cabinet Office, Government of Japan, Annual Report on the Japanese Economy and Public Finance 2009, 2009.

[7] Statistics Bureau of Japan, Family Income and Expenditure Survey, 2015.

[8] T. Piketty, Capital in 21th Century, Éditions du Seuil, 2013 (in French).
[9] E. Bonabeau, G. Theraulaz, J.-L. Deneubourg, Physica A 217, 373 (1995).

[10] R. Fujie, T. Odagaki, J. Phys. Soc. Jpn. 80, 124802 (2011).

[11] T. Odagaki, M. Tsujiguchi, Physica A 367, 435 (2006).

[12] M. Tsujiguchi, T. Odagaki, Physica A 375, 317 (2007).

[13] T. Okubo, T. Odagaki, Phys. Rev. E 76, 036105 (2007).

[14] R. Fujie, T. Odagaki, Physica A 389, 1471 (2010).

[15] Y. Todate, R. Fujie, T. Odagaki, Acta Phys. Pol. A 129, 937 (2016).

[16] D. Stauffer, Int. J. Mod. Phys. C 14, 237 (2003).

[17] E. Ben-Naim, S. Redner, J. Sat. Mech. Theory Exp. 2005, L11002 (2005).

[18] T.C. Schelling, J. Math. Soc. 1, 143 (1971). 